\title{
Occurrence of anti-D alloantibodies among pregnant women in Kasese District, Western Uganda
}

\section{Yona Mbalibulha' \\ Enoch Muwanguzi' \\ Godfrey R Mugyenyi² \\ Bernard Natukunda' \\ 'Department of Medical Laboratory Sciences, ${ }^{2}$ Department of Obstetrics and Gynecology, Faculty of Medicine, Mbarara University of Science and Technology, Mbarara, Uganda}

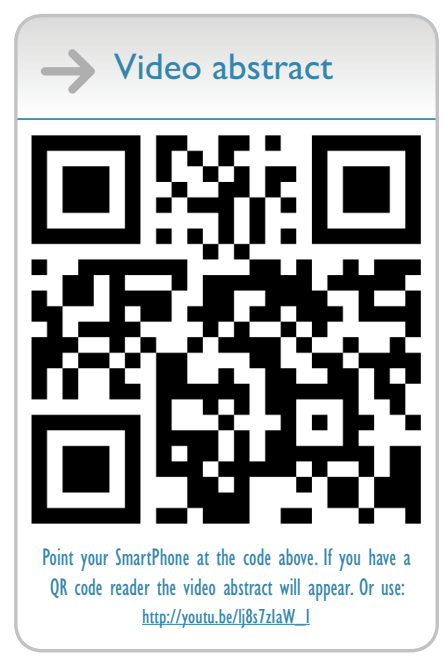

Correspondence: Yona Mbalibulha Department of Medical Laboratory Sciences, Faculty of Medicine, Mbarara University of Science and Technology, PO Box I4I0, Mbarara, Uganda Tel +256752454242

Email yonamilly@yahoo.co.uk
This article was published in the following Dove Press journal: Journal of Blood Medicine

17 April 2015

Number of times this article has been viewed

Objectives: This study was undertaken to determine the distribution of $\mathrm{ABO} / \mathrm{RhD}$ (rhesus $\mathrm{D}$ antigen) blood phenotypes, prevalence of anti-D alloantibodies, and the risk factors for alloimmunization among pregnant women in Kasese District, Western Uganda.

Materials and methods: Ethylenediamine tetraacetic acid-containing plasma samples and serum samples were taken from pregnant women attending the antenatal clinic. The blood groups were identified using the microplate grouping method, while the presence of anti-D alloantibodies was detected by the indirect antiglobulin test (IAT). Data were also collected from the pregnant women on the risk factors associated with anti-D alloantibody formation.

Results: Among the 726 participants, the blood group distribution was as follows: O: 356 (49.\%); A: 190 (26.\%); B: 152 (21\%); and AB: 28 (4\%). A total of 28 (3.86\%) pregnant women were RhD negative. Anti-D alloantibodies were detected in $88(12.1 \%)$ of the participants; and of these, 13 (14.8\%) were RhD negative. Statistically significant risk factors for anti-D alloimmunization included miscarriage, stillbirth, and postpartum hemorrhage.

Conclusion: Blood group $\mathrm{O}$ was the most common among the pregnant women in this study and the prevalence of Rh negativity was 3.8\%. The frequency of anti-D alloimmunization among pregnant women in Kasese District was $12.12 \%$, with $85.5 \%$ of these being RhD positive. Risk factors such as a history of stillbirths, miscarriages, and incidence of postpartum hemorrhage were significantly associated with anti-D alloimmunization. There is a need to routinely carry out antenatal blood grouping and IAT screening on pregnant women in Uganda to detect anti-D alloimmunization. Given the high prevalence of anti-D alloantibody formation among RhDpositive women, we recommend additional research studies on the role of autoimmunity among antigen-positive women, as well as the occurrence of $\mathrm{RhD}$ variants plus their implications on hemolytic disease of the fetus and newborn, in Uganda.

Keywords: $\mathrm{ABO} / \mathrm{RhD}$ blood groups, anti-D alloimmunization, indirect antiglobulin test, pregnant women, Western Uganda

\section{Introduction}

There are approximately 400 red blood cell antigens in 33 blood group systems. ${ }^{1}$ Of these, $>50$ different antigens are capable of causing maternal alloimmunization and fetal hemolytic disease. Rhesus $\mathrm{D}$ antigen ( $\mathrm{RhD}$ ) alloimmunization has been a focus of concern for obstetricians and hematologists for centuries because of the occurrence of $\mathrm{Rh}$ hemolytic disease. The prevalence of $\mathrm{RhD}$ negative group varies in different ethnic populations, with approximately $15.8 \%$ of Caucasians, $8 \%$ of Blacks, and $1 \%$ of Asians being RhD negative. ${ }^{2}$ Exposure to the $\mathrm{D}$ antigen is vital in obstetrics because if the maternal anti-D is immunoglobulin $\mathrm{G}(\operatorname{IgG})$, it is capable of crossing the placenta and causing complications such as hemolytic disease of the fetus and the newborn 
(HDFN). HDFN can present in three forms based on the red cell antigens to which maternal immunization has occurred; these include the following: 1) HDFN due to ABO antigens, 2) HDFN due to Rh, either due to anti-D alone or due to anti-D accompanied by other Rh antibodies such as anti-C, anti-c, anti-E, or anti-e, and 3) HDFN caused by "Other" factors - unexpected immune antibodies other than anti-D, which may be Jk, K, Fy, and S. The HDFN toward or due to $\mathrm{Rh}$ is the most common and severe. ${ }^{3}$

Although most people are either D+ or D-, there is a plethora of D variants, often categorized as either weak D or partial D. DVI is the D variant most commonly associated with anti-D production. Weak D types 1,2 , and 3 are rarely associated with alloanti-D production, so a policy recommendation would be to treat patients with those $\mathrm{D}$ variants as D+, patients with all other D variants would be treated as D-. ${ }^{4}$ These variants can lead to alloimmunization in RhDpositive pregnant women and the proportion of these variants is not established in Uganda.

Routine antenatal RhD and alloanti-D screening among women is a tool used in identifying significant maternal risk factors putting the neonate at risk of HDFN. This should then be followed by the administration of anti-D prophylaxis dose antepartum at the 28th week of gestation and a postpartum injection of anti-D within 72 hours of delivering an RhDpositive infant. ${ }^{5,6}$ However, in Uganda, there is no routine screening for maternal alloimmunization. Hence, we carried out this study to assess the level of alloimmunization within pregnant women in western Uganda.

\section{Materials and methods Study participants}

A total of 726 pregnant women attending the antenatal clinic at the Kagando Hospital, Bwera Hospital, and Nyabirongo Health Centre IV in Kasese District, Western Uganda, were recruited into the study. Participants consented and the study was approved by the research and ethics committee of Mbarara University and by the Uganda National Council of Science and Technology.

\section{Sample collection and analysis}

Whole blood ( $4 \mathrm{~mL}$ ) was drawn by venipuncture of the antecubital vein into plain and ethylenediaminetetraacetic acidcontaining vacutainer tubes. Samples were analyzed for ABO and $\mathrm{RhD}$ blood groups using the microplate method after dilution of red blood cells to a 5\% suspension with $0.9 \%$ $(\mathrm{w} / \mathrm{v})$ saline and adding the grouping antisera appropriately. These were then vortexed, and the presence or absence of agglutination was checked for. The indirect antiglobulin test (IAT) was performed, which involved the incubation of standard cells with the test serum at $37^{\circ} \mathrm{C}$ for 30 minutes and then reacting with anti-human globulin to bring about agglutination of the red cells in positive samples.

\section{Statistical analysis}

The information was recorded using data collection forms. Data were then entered using the statistical software package EXCEL 5.0 (Microsoft, Redmond, WA, USA) and transferred to STATA 12 (StataCorp, College Station, TX, USA) for analysis. Descriptive statistics (mean, median, interquartile range, and standard deviation) were used and the proportions calculated for categorical variables. Categorical data were analyzed using bivariate and multivariate comparisons. The $P$-value was set at $\leq 0.05$ to be considered statistically significant.

\section{Results \\ Demographic characteristics of the participants}

The participants had an age range of 15-42 (median: 24, IQR: 20, 30) years and there were 160 (22\%) prime gravid women. Complications reported in the present pregnancy included malaria, bleeding, and HIV. A total of 170 women (23.4\%) had a previous obstetric history of abortion (14\%), cesarian section $(2.75 \%)$, stillbirths $(5.65 \%)$, and postpartum hemorrhage (PPH) (0.96\%). Nine women (1.24\%) had a history of blood transfusion.

\section{Blood group distribution and maternal alloimmunization}

Most of the participants had blood group O $(356 ; 49.04 \%)$, with women of other blood groups as follows: A: $190(26 \%)$, B: $152(21 \%)$, and AB: $28(3.86 \%)$. A total of $28(3.86 \%)$ participants were $\mathrm{RhD}$ negative. There were 88 (12.1\%) IAT-positive participants, and of these, 13 (14.8\%) were of RhD-negative status and 75 (85.2\%) were RhD positive.

\section{Risk factors for anti-D alloimmunization}

There was an association of obstetric history, parity, and gravidity with alloimmunization. Thus, $43 \%$ had a parity of two to four children, while $37.5 \%$ had a parity of more than four children; the remaining participants were prime gravid women. A bivariate analysis of risk factors closely associated with $\mathrm{RhD}$ alloimmunization showed that obstetric history, age, and parity/gravidity had $P$-values of $0.001,0.025$, and 0.114 , respectively, thus being significantly associated 
with alloimmunization. In all the pregnant women, a positive association was common with obstetric history ( $P$-value: $0.0001)$ and transfusion history ( $P$-value: 0.2966$)$. There was no association with factors such as HIV and cesarian section generally. Factors such as abortion/miscarriage, stillbirth, and PPH in obstetric history had a close association. Age category ( $>30$ years) showed a significant association with anti-D alloimmunization ( $P$-value: 0.004$)$ (Tables 1 and 2$)$.

\section{Discussion}

$\mathrm{RhD}$ alloimmunization has been a focus of concern for obstetricians and hematologists for centuries in their quest to try and eliminate HDFN as a common obstetric problem. The alloanti-D antibodies arise following sensitizing factors such as pregnancy when a $\mathrm{RhD}$-negative woman conceives a $\mathrm{RhD}$-positive fetus, transfusion of $\mathrm{RhD}$-positive blood to RhD-negative woman, and obstetric sensitizing factors such as abortion, ectopic pregnancy, and hemorrhagic episodes in $\mathrm{RhD}$-positive women, and this phenomenon has remained a problem leading to HDFN. ${ }^{7}$ In this study, the focus was on both RhD-positive and RhD-negative pregnant women; we wanted to determine the ABO blood group distribution, test for the presence of alloantibodies, and establish the associated risks.

Table I Risk factors for maternal anti-D alloimmunization

\begin{tabular}{|c|c|c|}
\hline Variable & OR $(95 \% \mathrm{Cl})$ & $P$-value \\
\hline \multicolumn{3}{|l|}{ Age in years* } \\
\hline$\geq 30$ & $1.74(1.08-2.80)$ & 0.02 \\
\hline \multicolumn{3}{|l|}{ Parity/gravidity } \\
\hline Prime gravid & $0.98(0.53-1.80)$ & 0.11 \\
\hline Para $>1$ & $1.63(0.87-3.05)$ & \\
\hline \multicolumn{3}{|l|}{ Trimester } \\
\hline Second & $0.833(0.50-1.37)$ & 0.71 \\
\hline Third & $0.790(0.42-|.5|)$ & \\
\hline \multicolumn{3}{|l|}{ Pregnancy complications } \\
\hline Malaria & $1.24(0.50-3.06)$ & 0.89 \\
\hline Bleeding & $0.91(0.11-7.37)$ & \\
\hline \multicolumn{3}{|c|}{ Obstetric history of immunizing events } \\
\hline Abortion & $3.11(1.81-5.35)$ & 0.00 \\
\hline Stillbirths & $3.71(1.75-7.85)$ & \\
\hline $\mathrm{PPH}$ & $7.59(1.65-34.87)$ & \\
\hline \multicolumn{3}{|l|}{ Blood transfusion history } \\
\hline One transfusion episode & $2.09(0.42-10.25)$ & 0.39 \\
\hline Variable & COR $(95 \% \mathrm{Cl})$ & $P$-value \\
\hline \multicolumn{3}{|l|}{ Obstetric history } \\
\hline Abortion/miscarriage & $2.90(1.63-5.19)$ & 0.000 \\
\hline Stillbirth & $3.35(1.50-7.48)$ & 0.003 \\
\hline PPH & $8.94(1.93-41.27)$ & 0.005 \\
\hline
\end{tabular}

Note: $* \mathrm{~N}=724$.

Abbreviations: $\mathrm{Cl}$, confidence interval; $\mathrm{COR}$, crude odds ratio; OR, odds ratio; $\mathrm{PPH}$, postpartum hemorrhage.
Table 2 Risk factors of alloimmunization in sensitized and nonsensitized pregnant women $(\mathrm{N}=726)$

\begin{tabular}{|c|c|c|c|}
\hline \multirow[t]{2}{*}{ Variable } & \multicolumn{2}{|l|}{ n (\%) } & \multirow[t]{2}{*}{$P$-value } \\
\hline & $\begin{array}{l}\text { IAT positive } \\
(\mathrm{n}=\mathbf{8 8})\end{array}$ & $\begin{array}{l}\text { IAT negative } \\
(\mathrm{n}=638)\end{array}$ & \\
\hline \multicolumn{4}{|l|}{ Age in years* } \\
\hline$<30$ & $57(64.77)$ & $485(76.26)$ & \multirow[t]{2}{*}{0.025} \\
\hline$\geq 30$ & $31(35.23)$ & 151 (23.74) & \\
\hline \multicolumn{4}{|l|}{ Parity/gravidity } \\
\hline Prime gravid & $17(19.32)$ & | $43(22.4 \mid)$ & \multirow[t]{3}{*}{0.114} \\
\hline Para 2-4 & $38(43.18)$ & $325(50.94)$ & \\
\hline Para $>4$ & $33(37.50)$ & $170(26.65)$ & \\
\hline \multicolumn{4}{|l|}{ Trimester } \\
\hline First & $32(36.36)$ & $204(31.97)$ & \multirow[t]{3}{*}{0.721} \\
\hline Second & $40(11.56)$ & $306(47.96)$ & \\
\hline Third & $16(18.18)$ & $128(20.06)$ & \\
\hline \multicolumn{4}{|l|}{ Pregnancy complications } \\
\hline Malaria & $6(6.82)$ & $35(5.49)$ & \multirow[t]{3}{*}{0.923} \\
\hline Bleeding & I (I.I4) & $9(1.25)$ & \\
\hline HIV & $0(0.00)$ & $5(0.63)$ & \\
\hline \multicolumn{4}{|l|}{ Obstetric history } \\
\hline Abortion/miscarriage & $24(27.27)$ & $78(12.23)$ & \multirow[t]{4}{*}{0.000} \\
\hline Cesarian section & $0(0.00)$ & $20(3.13)$ & \\
\hline Stillbirth & II (12.50) & $30(4.70)$ & \\
\hline $\mathrm{PPH}$ & $3(3.4 I)$ & $4(0.63)$ & \\
\hline Transfusion history & $2(2.27)$ & $7(1.10)$ & 0.299 \\
\hline \multicolumn{4}{|l|}{ Transfusion episode } \\
\hline One & $2(2.27)$ & $7(1.10)$ & 0.299 \\
\hline
\end{tabular}

Note: $* \mathrm{~N}=724$.

Abbreviations: HIV, human immunodeficiency virus; IAT, indirect antiglobulin test; $\mathrm{PPH}$, postpartum hemorrhage.

Our study established a distribution of the ABO blood group, with group $\mathrm{O}(49 \%)$ having the highest percentage, followed by A (26\%), B (20.9\%), and AB (3.9\%). This is similar to the result of the study carried out in Kampala, Uganda, where the blood group distribution was $48.9 \%$, $26.4 \%, 21.0 \%$, and $3.8 \%$ for the blood groups $\mathrm{O}, \mathrm{A}, \mathrm{B}$, and $\mathrm{AB}$, respectively, ${ }^{8}$ but differing greatly from the distribution among the Loe population of the Lao Republic, with distributions as follows: O 37.7\%, B 35.6\%, A 19.9\%, and AB 6.0\%. This is because of the ethnic variation.

In this study, $\mathrm{Rh}$ group distribution was $3.86 \%$; which is similar to a study that was carried out in southwestern Uganda and which showed 3.6\%. ${ }^{9}$ But the Rh distribution is lower than the $7.1 \%$ found in Black blood donor populations in the USA and $7.5 \%$ found in Saudi Arabia. ${ }^{10}$ We observed a lower prevalence of $\mathrm{RhD}$-negative status (4.0\%) among the pregnant women studied herein compared to that among Caucasians ( $\geq 14 \%)$. Our findings are consistent with several previous studies among Africans and Asians, reporting $\mathrm{RhD}$ prevalence of $\leq 7.2 \%{ }^{11}$

A total of $88(12.12 \%)$ participants tested positive, indicating the presence of the antibodies. This indicated a higher 
prevalence of these antibodies in relation to the results of a similar study ${ }^{8}$ conducted in Kampala, wherein a prevalence of $5.5 \%$ was obtained. Among the 88 participants who were IAT positive, $75(85.2 \%)$ were $\mathrm{RhD}$-positive pregnant women, indicating the development of alloantibodies within the RhD-positive women, which could mainly be due to $\mathrm{RhD}$ variants within the population or, to some extent, due to autoimmunity triggered by anti-RhD antibody coating the erythrocytes, and this need to be verified further.

It is possible that not all the women in the study could recall being transfused in early childhood, which may explain the presence of alloantibodies in the prime gravid women and the absence of previous transfusion as a risk factor for maternal alloimmunization. However, the alloimmunization frequency in our teenage pregnant women was comparable to the frequency, albeit greater, in older women. It is possible to speculate that persistence of an antibody formed after an unrecognized transfusion during childhood explains antibody prevalence in the young women, while high parity is responsible for antibody prevalence in the older women. This trend was also observed in other Ugandan studies. ${ }^{8,9}$

Age was significantly associated with the rate of alloimmunization $(P=0.0007)$ in $\mathrm{RhD}$-negative pregnant women. Because parity rises with age, the rate of alloimmunization might rise with maternal age; this is because the association was found in those aged $\geq 30$ years because these women are continuously exposed to risk factors during every pregnancy. The alloimmunization in the young women could be explained by other sensitizing factors, such as childhood blood transfusion, which could not be remembered. This is comparable to a study in southwestern Uganda, where several sensitizing factors such as parity, age, and other obstetric factors were found to be associated with alloimmunization. ${ }^{9}$

In the RhD-positive pregnant women, a strong association was obtained with the obstetric history $(P=0.0001)$. It is therefore indicative that risk factors such as miscarriage, stillbirth, and PPH ( $P=0.001,0.002$, and 0.012 , respectively) play a key role in $\mathrm{RhD}$ alloimmunization in $\mathrm{RhD}$-positive pregnant women as in the RhD-negative women. Other factors such as transfusion history and having blood group $\mathrm{O}$ had a mild association, but this was not strong enough to be strongly linked. This could be that few pregnant women report having been transfused or could not remember.

\section{Conclusion}

Blood group O-positive is the most prevalent blood group among pregnant women attending antenatal clinics in Kasese District and the prevalence of $\mathrm{Rh}$ negativity was $3.8 \%$.
The prevalence of $\mathrm{Rh}$ alloimmunization in pregnant women was found at $12.12 \%$, with $85 \%$ of these women being $\mathrm{RhD}$ positive, which is higher than that reported in similar studies in Kampala. Risk factors such as stillbirth, miscarriage/abortion, and PPH had a close association with alloimmunization.

The implications of our findings are limited given the deidentified nature of the specimens tested and lack of follow-up data on the newborns of these women. Consequently, this study only showed the presence of demonstrable alloantibody, but it would be necessary to establish the frequency and specificities of demonstrable clinically significant antibodies that may cause HDFN or require compatible blood for transfusion among pregnant women and their neonates in Kasese. It demonstrates the feasibility of establishing the blood group type and screen testing for $\mathrm{RhD}$ alloantibody in a health care setting.

\section{Acknowledgments}

The authors wish to thank the study participants. We are grateful to the Medical Education Partnership InitiativeMedical Education Services for All Ugandans (MEPIMESAU) consortium.

\section{Funding}

This work was supported by grant number 5R24TW008886, supported by Office of the US Global Aids Coordinator, National Institutes of Health, and Health Resources and Services Administration. Its contents are solely the responsibility of the authors and do not necessarily represent the official views of the supporting offices.

\section{Author contributions}

YM participated in study conception and design, data acquisition, analysis and interpretation, manuscript drafting and revising. EM participated in data collection, analysis and interpretation, manuscript drafting and revising. GM participated in study design, data analysis and interpretation, manuscript drafting and revising. BN participated in study conception, design, data analysis and interpretation, manuscript drafting and revising.

\section{Disclosure}

The authors report no conflicts of interest in this work.

\section{References}

1. John G. Wintrobe's Clinical Haematology. Vol 1. 11th ed. Netherlands: Wolters Kluwer; 2008:804-805.

2. Avent ND, Reid ME. The Rhesus blood group system. J Am Soc Haematol. 2000;95(2):375-387. 
3. Catrina FR. Hemolytic disease of of the new born. REX Lab Bulletin. 2007;126:1-4

4. Daniels G. Variants of RhD current testing and clinical consequences. Br J Haematol. 2013;161(4):461-470.

5. Gajjar K, Spencer C. Diagnosis and management of non anti-D red cell antibodies in pregnancy. Obstetr Gynaecol. 2009;11:89-95.

6. Kumar S, Regan F. Management of pregnancies with RhD alloimmunisation. Br Med J. 2005;330:1255-1258.

7. Marek L. Prevention of alloimmunisation in RhD negative women. Biomed Pap MeFac Univ Palacky Olomouc Czech Repub. 2010;1(154):3-8.
8. Eipl K. Seroprevalence of unexpected red cell antibodies among pregnant women in Uganda. Immunohematology. 2012;4(28):115-117.

9. Natukunda B, Mugenyi G, Brand A, Schonewille H. Maternal red blood cells alloimmunisation in south western Uganda. Transfus Med. 2011;21(4):262-266.

10. Bondagji NS. Rhesus alloimmunization in pregnancy. A tertiary care center experience in the Western region of Saudi Arabia. Saudi Med J. 2012;33(6):688

11. Erhabor O, TC A. Rh alloimmunization in Sub Saharan Africa. Int $J$ Womens Health. 2010;2:429-437.
Journal of Blood Medicine

\section{Publish your work in this journal}

The Journal of Blood Medicine is an international, peer-reviewed, open access, online journal publishing laboratory, experimental and clinical aspects of all topics pertaining to blood based medicine including but not limited to: Transfusion Medicine; Blood collection, Donor issues, Transmittable diseases, and Blood banking logistics; Immunohematology; Artificial and alternative

\section{Dovepress}

blood based therapeutics; Hematology; Biotechnology/nanotechnology of blood related medicine; Legal aspects of blood medicine; Historical perspectives. The manuscript management system is completely online and includes a very quick and fair peer-review system. Visit http://www.dovepress.com/ testimonials.php to read real quotes from published authors.

Submit your manuscript here: http://www.dovepress.com/Journal-of-blood-medicine-journal 